\title{
Anomalie congenite dei reni e delle vie urinarie (CAKUT)
}

\author{
Francesca Liut ${ }^{1 *},{\text { Claudia } \text { Izzi }^{1} \text {, Nadia Dallera }}^{1}$, Francesco Scolari ${ }^{1,2}$ \\ ${ }^{1}$ Divisione di Nefrologia e Dialisi, Ospedale di Montichiari, Montichiari (BS) \\ ${ }^{2}$ Cattedra di Nefrologia, Università di Brescia, Brescia \\ ${ }^{*}$ Affiliazione attuale: ASST Franciacorta, P.O. Chiari Servizio Emodialisi, Chiari (BS)
}

\begin{abstract}
Congenital Anomalies of the Kidney and the Urinary Tract (CAKUT)
Congenital Anomalies of the Kidneys and the Urinary Tract (CAKUT) involves several morphological anormalies of the kidneys and the urinary tract directly linked to congenital defects of kidney embryogenesis.

It represents about $23 \%$ of all congenital defects, $50 \%$ of End-Stage Renal Disease (ESRD) in pediatric patients and $2-7 \%$ of ESRD in adult patients.

CAKUT includes a wide spectrum of morphological anomalies of the kidneys and the urinary tract with heterogeneous case histories and modalities of transmission from severe to familiar ones, sporadic, autosomal recessive or closely linked to de novo mutations.

Environmental factors can also be involved as clearly highlighted by the association between pregnancy-related diabetes mellitus and renal agenesia and/or alcohol or cocaine abuse.

CAKUT pathogenesis is multifactorial but renin-angiotensin system encoding gene mutations seems to be involved in CAKUT development.

Only $10 \%$ of all CAKUT patients present diagnosed genetic mutations at present time.

Due to the wide spectrum of disease's onset and the different impact of renal anomalies, a clinical approach tailored to every single patient with an extended follow-up period and an accurate genetic counseling is mandatory.
\end{abstract}

Keywords: CAKUT, Congenital anomalies, Genetic counseling, Renal embryogenesis

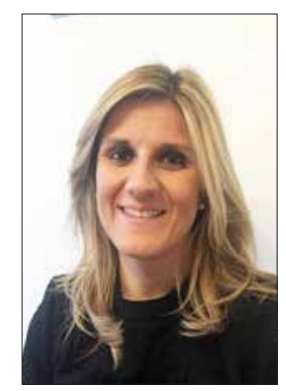

Francesca Liut

\section{Introduzione}

Le Anomalie congenite dei reni e delle vie urinarie (Congenital Anomalies of the Kidneys and the Urinary Tract - CAKUT) configura un gruppo di anomalie morfologiche a carico di rene e vie escretrici associate a difetti dell'embriogenesi renale. II CAKUT rappresenta il $23 \%$ di tutti i difetti identificati alla nascita; viene riscontrato in più dell' $1 \%$ dei nati vivi e rappresenta circa il $50 \%$ delle cause di End-Stage Renal Disease (ESRD) in età pediatrica, con elevata mortalità se si manifesta alla nascita o nel primo anno di vita $(1,2)$. Tale condizione comprende un ampio spettro di malformazioni a carico rispettivamente del rene (agenesia renale, displasia renale multicistica, displasia/

Accepted: March 25, 2016

Published online: May 4, 2016

Indirizzo per la corrispondenza:

Dr.ssa Francesca Liut

ASST Franciacorta, P.O. Chiari Servizio Emodialisi

Via Giuseppe Mazzini 4

25032 Chiari (BS)

franciliut@hotmail.it ipoplasia renale, rene a ferro di cavallo) e delle vie escretrici, come megauretere congenito, ectopia ureterale, duplicazioni del sistema escretore, stenosi del giunto uretero-vescicale, valvole uretrali posteriori, stenosi ostruttiva del giunto pieloureterale, ureterocele e reflusso vescico-ureterale (VUR). Tali anomalie possono essere presenti singolarmente o in varie combinazioni. Nell'adulto, rappresenta circa il 2-7\% delle cause di ESRD, ma questo dato è spesso sottostimato; infatti, molti pazienti affetti da CAKUT risultano asintomatici nell'infanzia e possono manifestare la patologia progressivamente in età adulta (3).

\section{Eziologia e geni coinvolti}

La sua eziologia è da ricercare nell'embriogenesi renale ed è associata a mutazioni di geni che regolano pathway cellulari coinvolti nello sviluppo renale e dell'asse escretore; si tratta, infatti, di un processo complesso che prende origine a partire dalla quinta settimana di sviluppo nell'uomo: il dotto nefrico viene a costituirsi dal mesoderma intermedio e si estende caudalmente, inducendo il mesoderma adiacente a dare origine al pronefro e al mesonefro. Il mesonefro dà origine agli organi riproduttivi maschili. Al $28^{\circ}$ giorno, il dotto nefrico dà origine a un'ulteriore struttura chiamata gemma ureterale, che invade il mesenchima metanefrico adiacente, 
inducendo la formazione del rene metanefrico permanente. La porzione distale della gemma ureterale darà origine al sistema collettore renale, mentre il mesenchima metanefrico darà origine alle cellule epiteliali che costituiranno i nefroni (4). II CAKUT rappresenta un gruppo piuttosto eterogeneo di malattie con differenti presentazioni e modalità di trasmissione. Si identificano forme severe, con mortalità perinatale molto elevata, forme familiari, con modalità di trasmissione soprattutto autosomica dominante a penetranza incompleta, generalmente associate a mutazioni determinanti "loss of function" o alleli ipomorfi, che danno origine a fenotipi mild della malattia, e forme sporadiche, che rappresentano la maggior parte dei casi e che spesso sono caratterizzate da una trasmissione autosomica recessiva o legate a mutazioni de novo. A volte, il CAKUT si manifesta nell'ambito di sindromi in cui alle anomalie renali si associano malformazioni vertebrali e cardiache, atresia anale e fistole tracheo-esofagee (5).

\section{Forme sindromiche}

In numerosi modelli sperimentali in vivo è stato dimostrato che le forme sindromiche di CAKUT possono essere determinate da un difetto di un singolo gene. Esse rappresentano circa il $10 \%$ di tutte le forme di CAKUT e possono essere distinte in forme a trasmissione autosomica dominante a penetranza incompleta oppure in forme recessive. Nel 1995, venne, per la prima volta, descritta la mutazione puntiforme a carico del gene PAX2 in una famiglia affetta da coloboma del nervo ottico, in cui erano presenti anche ipoplasia renale e VUR; successivamente, un secondo gene, HNF1B (TCF2) venne correlato al CAKUT per la scoperta di una mutazione eterozigote in due fratelli con diabete e cisti renali (6). In uno studio di Weber et al, condotto in pazienti con ipodisplasia renale, è stato testato il ruolo di mutazioni a carico di geni implicati nella morfogenesi renale, tra cui TCF2, PAX2, EYA1, SIX1 e SALL; questo studio mostrava che le varianti deleterie di TCF2 e PAX2 risultavano essere le più frequenti in questi pazienti e veniva, pertanto, ipotizzato che avessero un ruolo predominante nella genesi del CAKUT, soprattutto per quanto riguarda le forme monogeniche. In particolare, mutazioni di PAX2 erano associate a displasia o a ipoplasia renale, mentre mutazioni a carico di HNF1B erano più frequentemente associate $a$ cisti renali (7). Recentemente, è stato scoperto che, a carico del gene DSTYK (dual serine-threonine and tyrosine protein kinase), che codifica per una chinasi a doppia specificità per serina/treonina e tirosina, venivano riscontrate mutazioni nel $2,3 \%$ dei pazienti portatori di malformazioni renali e delle vie urinarie; sembra, infatti, che tale gene regoli la via di segnalazione del fibroblast growth factor (FGF) nel corso dello sviluppo delle vie urinarie (8). Le forme monogeniche sindromiche di CAKUT, caratterizzate da una mutazione recessiva, rappresentano una frazione esigua (inferiore al $2 \%$ ) ma risultano avere una più chiara correlazio- ne genotipo-fenotipo, a differenza di quelle a trasmissione autosomica dominante, probabilmente per una penetranza incompleta o per una variabilità di espressione, oltre a un possibile ruolo dei geni modificatori (9).

\section{Eterogeneità genetica}

Nella maggior parte dei casi, il CAKUT presenta un'importante eterogeneità genetica, con un'espressione variabile del fenotipo e una bassa correlazione genotipo-fenotipo, probabilmente dovuta a modelli complessi di ereditarietà che coinvolgono più geni in loci multipli. Sono stati, per esempio, riportati casi di contemporanea presenza di mutazioni a carico di PKD1 e di altri cistogeni, in particolare di HNF1B, in alcuni membri di famiglie con malattie cistiche renali; $i$ pazienti portatori di varianti germinali in due cistogeni presentavano un esordio più precoce e più grave della malattia (10). Un altro fattore di variabilità genica sembra essere associato alla presenza di copy number variations (CNV): esse sono definite come qualsiasi aumento o perdita di DNA germinativo di dimensioni comprese tra 1 kilobase $(\mathrm{kb})$ e svariate megabasi ( $\mathrm{Mb}$ ). Queste possono essere ricorrenti, se sono generate mediante ricombinazione non allelica, o non ricorrenti, se i punti di rottura sono diversi in ogni singolo individuo. In uno studio effettuato da Sanna-Cherchi et al su 522 bambini con agenesia renale o ipodisplasia renale, veniva dimostrata la presenza di un maggior carico mutazionale nei casi che nei controlli e che le malformazioni renali si associavano a CNV di dimensioni uguali o inferiori a $250 \mathrm{~kb}$ in circa il $17 \%$ dei casi (11). Tali anomalie non sono facilmente diagnosticabili con tecniche di citogenetica convenzionale e risultano essere una causa frequente di CAKUT. Spesso si associano, inoltre, a fenotipi di sviluppo neurocognitivo. La nefrogenesi, pertanto, risulta essere sensibile alla variazione del dosaggio genico.

\section{Fattori ambientali}

Anche i fattori ambientali sono direttamente correlati alla storia naturale della malattia; è nota, infatti, l'associazione tra diabete mellito pre-gestazionale materno e agenesia renale, come rilevato dallo studio caso-controllo effettuato in Colorado su 189 bambini con agenesia renale $(O R=4.98$; $95 \% \mathrm{Cl} 1.08-22.93$ ) (12); è stato, inoltre, documentato che l'esposizione precoce a diabete mellito aumentava il rischio di CAKUT (OR = 1.67; 95\% Cl 1.14-2.46) (13). Infine, bisogna segnalare che è stato dimostrato che l'esposizione ad alcol o alla cocaina durante la gravidanza può essere associata a CAKUT nei bambini.

\section{Fattori epigenetici}

I fattori epigenetici risultano essere cruciali nella regolazione dell'espressione genica (epigenetic programming). 
I meccanismi epigenetici più studiati sono rappresentati dall'acetilazione, dalla metilazione o dalla fosforilazione degli istoni e dalla metilazione della cromatina. Questi processi possono modulare la struttura della cromatina, per esempio favorendo il passaggio da etero cromatina, forma inattiva e compatta, a eucromatina, forma aperta, accessibile ai fattori di trascrizione, in grado di facilitare l'espressione o il silenziamento di alcune regioni del genoma. Uno studio di Patel et al (14) ha dimostrato che esiste un legame tra PAX2 e metilazione della cromatina. I fattori epigenetici risultano suscettibili allo stress ambientale e determinano una variabilità nel pattern di espressione genica; possono, per esempio, influenzare lo sviluppo di pathway pro-fibrotici. Jin et al (15) hanno dimostrato, in due gemelli omozigoti con agenesia renale discordante, che erano presenti, a livello genico, 514 differenti regioni metilate, mentre non si riscontravano differenze nelle CNV, motivo per cui i fattori epigenetici concorrevano direttamente alla patogenesi di CAKUT. È stato rilevato, inoltre, che anche mutazioni a carico dei geni che codificano per mediatori del sistema renina-angiotensina (RAS) possono avere un ruolo nella patogenesi di CAKUT; infatti, in uno studio effettuato nel 2005 in bambini francesi, tali mutazioni determinavano una forma molto grave di displasia tubulare renale associata a oligo-idramnios; a livello corticale renale, si osservava una riduzione del numero di tubuli prossimali, mentre, a livello midollare, erano presenti una cospicua fibrosi e dotti collettori collassati; i piccoli pazienti presentavano ipotensione e la maggior parte moriva in fase perinatale per anuria associata a ipoplasia polmonare (16).

\section{Tecniche genetiche per lo studio dei geni candidati}

La scoperta di geni causali risulta essere più agevole per le forme sindromiche di CAKUT rispetto alle forme non-sindromiche. Lo screening di geni candidati, tra cui BMP7, CHD1L, CDC5L, EYA1, GATA3, RET, ROBO2, SALL1, SIX2, SIX5 e altri, può dare un risultato in circa il 6-20\% dei casi nei pazienti CAKUT con mutazioni in eterozigosi. La maggior parte degli studi non riporta, però, l'analisi di co-segregazione. Sono stati attivati dei database (per esempio, Exome Aggregation Consortium) che possono fungere da riferimento per le varianti già identificate. L'utilizzo di analisi di linkage risulta essere utile nel caso di forme mendeliane ed è stata utilizzato con buoni risultati per il VUR familiare in una famiglia somala con 12 figli affetti da CAKUT e in 7 pedigree di famiglie italiane affette da ipodisplasia renale. Tali analisi, però, non risultano essere completamente esaustive in corso di CAKUT, poiché spesso si tratta di condizioni con penetranza familiare incompleta. Talvolta, l'analisi di linkage può, inoltre, rilevare difetti genetici a livello di regioni non-coding, che devono essere caratterizzate meglio con altre metodiche. A oggi, è stato dimostrato che meno del $10 \%$ dei pazienti affetti da
CAKUT è portatore di mutazioni a carico dei geni noti; pertanto, la maggior parte delle cause di CAKUT non è ancora nota. Sono stati identificati recentemente nuovi geni, tra cui ITGA8 e TRAP 1, responsabili di forme a trasmissione autosomica recessiva. L'identificazione, invece, di CNV è possibile grazie a microarray ad alta risoluzione, ossia array-CGH (array comparative genomic hybridization) e SNP microarray (single nucleotide polymorphism microarray), metodiche che presentano vantaggi rispettivamente per i costi e per la risoluzione genomica, soprattutto con array che possono identificare CNV fino a 500 bp (base pair). L'impiego sequenziale di genome-wide linkage analysis e di whole-exome sequencing ha permesso di identificare il gene DSTYK (17).

L'ampio spettro di presentazione e il differente impatto clinico delle anomalie renali presenti nelle forme di CAKUT richiedono una gestione clinica del paziente personalizzata; spesso la morbidità della malattia nelle forme mild si presenta solo in età adulta; pertanto, questi pazienti devono essere sottoposti a un follow-up continuativo attraverso il monitoraggio dei valori pressori, della funzione renale e della proteinuria; è, inoltre, indicato un adeguato counseling genetico che possa guidare il paziente, in particolare nelle forme familiari e de novo.

\section{Disclosures}

Financial support: No financial support was received for this submission. Conflict of interest: The authors have no conflict of interest.

\section{Bibliografia}

1. Loane M, Dolk H, Kelly A, et al. Paper 4: EUROCAT statistical monitoring: identification and investigation of ten year trends of congenital anomalies in Europe. Birth Defects Res A Clin Mol Teratol 2011;91(Suppl. 1):S31-43.

2. Ardissino G, Dacco V, Testa S, et al. Epidemiology of chronic renal failure in children: data from the ItalKid project. Pediatrics 2003;111:e382-7.

3. Sanna-Cherchi S, Ravani P, Corbani V, et al. Renal outcome in patients with congenital anomalies of the kidney and urinary tract. Kidney Int 2009;76:528-33.

4. Schedl A. Renal abnormalities and their developmental origin. Nat Rev Genet 2007;8:791-802.

5. Woolf AS, Winyard PJ. Molecular mechanisms of human embryogenesis: developmental pathogenesis of renal tract malformations. Pediatr Dev Pathol 2002;5:108-29.

6. Thomas R, Sanna-Cherchi S, Warady BA, Furth SL, Kaskel FJ, Gharavi AG. HNF1B and PAX2 mutations are a common cause of renal hypodysplasia in the CKiD cohort. Pediatr Nephrol 2011;26:897-903.

7. Weber S, Moriniere V, Knuppel T, et al. Prevalence of mutations in renal developmental genes in children with renal hypodysplasia: results of the ESCAPE study. J Am Soc Nephrol 2006;17:2864-70.

8. Sanna-Cherchi S, Caridi G, Weng PL, et al. Localization of a gene for nonsyndromic renal hypodysplasia to chromosome 1p32-33. Am J Hum Genet 2007;80:539-49.

9. Weng PL, Sanna-Cherchi S, Hensle T, et al. A recessive gene for 
primary vesico-ureteral reflux maps to chromosome 12p11-q13. J Am Soc Nephrol 2009;20:1633-40.

10. Sanna-Cherchi S, Caridi G, Weng PL, et al. Genetic approaches to human renal agenesis/hypoplasia and dysplasia. Pediatr Nephrol 2007;22:1675-84.

11. Sanna-Cherchi S, Kiryluk K, Burgess KE, et al. Copy-number disorders are a common cause of congenital kidney malformations. Am J Hum Genet 2012;91:987-97.

12. Parikh CR, McCall D, Engelman C, et al. Congenital renal agenesis: case-control analysis of birth characteristics. Am J Kidney Dis 2002;39:689-94.

13. Dart AB, Ruth $C A$, Sellers EA, et al. Maternal diabetes mellitus and congenital anomalies of the kidney and urinary tract
(CAKUT) in the child. Am J Kidney Dis 2015;65:684-91.

14. Patel SR, Kim D, Levitan I, et al. The BRCT-domain containing protein PTIP links PAX2 to a histone $\mathrm{H} 3$, lysine 4 methyltransferase complex. Dev Cell 2007;13:580-92.

15. Jin M, Shida Z, Panpan H, et al. Genomic and epigenomic analyses of monozygotic twins discordant for congenital renal agenesis. Am J Kidney Dis 2014;64:119-22.

16. Gribouval O, Gonzales M, Neuhaus T, et al. Mutations in genes in the rennin-angiotensin system are associated with autosomal recessive renal tubular dysgenesis. Nat Genet 2005;37:964-8.

17. Sanna-Cherchi S, Sampogna RV, Papeta N, et al. Mutation in DSTYK and dominant urinary tract malformation. N Engl J Med 2013;369:621-9. 